\title{
Epidemiology, mortality and effectiveness of prophylaxis for Pneumocystis jiroveci pneumonia among rheumatic patients: a territory-wide study
}

\author{
Shirley Chiu Wai Chan, Ho Yin Chung, Chak Sing Lau and Philip Hei Li* (D)
}

\begin{abstract}
Background: Pneumocystis jiroveci pneumonia (PJP) is an opportunistic infection affecting immunocompromised individuals. However, evidence regarding the burden and effectiveness of prophylaxis among rheumatic patients remains limited. Delineating the epidemiology and efficacy of prophylaxis among rheumatic patients is urgently needed.
\end{abstract}

Methods: We performed a territory-wide cohort study of rheumatic patients in Hong Kong. All patients with a diagnosis of anti-neutrophil cytoplasmic antibody-associated vasculitis (AAV), immune-mediated myositis (IMM), rheumatoid arthritis (RA), systemic lupus erythematosus (SLE), systemic sclerosis (SSC), or spondyloarthritis (SpA) between 2015 and 2019 were included. Prevalence, frequency of prophylaxis and mortality of PJP were calculated. Number needed to treat (NNT) analysis was also performed.

Results: Out of 21,587 patients (54\% RA, 25\% SLE, 13\% SpA, 5\% IMM, 2\% AAV and 1\% SSc), 1141 (5.3\%) patients were prescribed PJP prophylaxis. 48/21,587 (0.2\%) developed PJP. No patients who developed PJP received prophylaxis prior to infection. The incidence of PJP was highest among SSC, AAV, and IMM patients. Among these diseases, the majority of PJP occurred while patients were on glucocorticoids at daily prednisolone-equivalent doses of $15 \mathrm{mg} /$ day (P15) or above. PJP prophylaxis was effective with NNT for SSc, AAV and IIM being 36, 48 and 114 respectively. There were 19 PJP-related mortalities and the mortality rate was 39.6\%.

Conclusion: PJP is an uncommon but important infection among rheumatic patients, PJP prophylaxis is effective and should be considered in patients with SSc, AAV and IMM, especially those receiving glucocorticoid doses above P15.

Keywords: Epidemiology, Prophylaxis, Rheumatology, Fungal, Pneumonia, Mortality

\section{Introduction}

Pneumocystis jiroveci pneumonia (PJP) is an opportunistic infection affecting immunocompromised individuals. Historically, PJP was the most common cause of death among patients with human immunodeficiency virus

\footnotetext{
*Correspondence: liphilip@hku.hk
}

Division of Rheumatology and Clinical Immunology, Department of Medicine, Queen Mary Hospital, The University of Hong Kong, Hong Kong, China
(HIV) but its incidence has declined over the past decades following the introduction of highly active antiretroviral therapy and routine PJP prophylaxis $[1,2]$. In contrast, PJP has become an increasingly important cause of atypical pneumonia among non-HIV immunocompromised patients due to the expanding armamentarium in immunosuppressive and chemotherapeutic therapies [3]. PJP in non-HIV patients runs an aggressive course and is associated with even higher mortality compared with original author(s) and the source, provide a link to the Creative Commons licence, and indicate if changes were made. The images or other third party material in this article are included in the article's Creative Commons licence, unless indicated otherwise in a credit line to the material. If material is not included in the article's Creative Commons licence and your intended use is not permitted by statutory regulation or exceeds the permitted use, you will need to obtain permission directly from the copyright holder. To view a copy of this licence, visit http://creativecommons.org/licenses/by/4.0/. The Creative Commons Public Domain Dedication waiver (http://creativeco mmons.org/publicdomain/zero/1.0/) applies to the data made available in this article, unless otherwise stated in a credit line to the data. 
HIV patients [4]. Due to its high mortality, PJP prophylaxis is commonly prescribed in many immunocompromising conditions and various guidelines have been established in oncology, bone marrow and solid organ transplant (SOT) [5-7]. Co-trimoxazole and aerolised pentamidine (especially for glucose-6-phosphate dehydrogenase deficient patients) are the preferred first-line agents for PJP prophylaxis in Hong Kong [8].

Patients with rheumatic diseases are at risk of PJP due to their immunocompromised state contributed by both the diseases and the use of immunosuppressive therapies. The risk of PJP varies across different rheumatic diseases. For example, it has been shown that granulomatosis with polyangiitis is associated with highest risk, while rheumatoid arthritis (RA) is associated with the lowest risk of PJP [9]. Use of immunosuppressive therapies, including glucocorticoids (GC) especially at high dose over a prolonged period of time and cyclophosphamide (CYC) have also been reported as risk factors for developing PJP [10]. Although some studies have demonstrated the efficacy and safety of PJP prophylaxis, these have been limited to only selected rheumatic diseases $[11,12]$. In comparison to other specialties, international consensus and guidelines are still lacking for rheumatic patients, largely due to absence of comprehensive observational studies to guide recommendations.

In order to address these shortcomings, we took advantage of Hong Kong's comprehensive electronic health record system and reviewed all rheumatic patient records of our territory-wide database. The Hospital Authority (HA) of Hong Kong has one of world's largest clinical information systems with a unified health record database for more than 7.1 million unique patients. Using this population-wide data, we performed a comprehensive study to delineate the incidence, mortality and efficacy of PJP prophylaxis in rheumatic patients of Hong Kong.

\section{Methods}

This was an observational, longitudinal cohort study based on data retrieved from the territory-wide electronic healthcare database (Clinical Data Analysis and Reporting System) of HA. The primary endpoint was the incidence of PJP in rheumatic patients. The secondary endpoint was the effectiveness of PJP prophylaxis. HA is the sole public-funded healthcare provider of Hong Kong and serves a population of over 7 million patients through 43 hospitals, 49 specialist out-patient clinics and 73 general out-patient clinics; covering around 90\% of all secondary and tertiary care of Hong Kong [12]. All data from this database is anonymized (having only identity numbers without patient contact details) to protect patient identity and has been extensively used for conducting other high-quality large big-data studies [13-16].
All patients with a diagnosis of anti-neutrophil cytoplasmic antibody-associated vasculitis (AAV), immunemediated myositis (IMM), rheumatoid arthritis (RA), systemic lupus erythematosus (SLE), systemic sclerosis (SSc), or spondyloarthritis (SpA) were included. All relevant longitudinal follow-up data from 1st January 2015 to 31st December 2019 was retrieved. Rheumatic diagnoses were defined based on diagnosis code using the International Classification of Diseases, Ninth Revision (ICD-9). Other essential clinical information including patients' demographics, medication prescriptions, blood tests and mortality (and cause, if any) were recorded. Medication prescriptions including the use of immunosuppressive agents within one year prior to PJP were analysed for patients with PJP. Blood tests included complete blood counts with differentials; liver and renal function tests at the time of PJP were evaluated.

PJP was defined as one of the following: ICD-9 diagnosis code and/or positive microbiological results from deep respiratory tract specimens including direct microbiological identification and/or detection by in-house quantitative polymerase chain reaction (PCR). Direct microbiological identification referred to the visualization of PJP cysts using methenamine silver stain. A positive PCR in the absence of clinical manifestations was not considered as PJP, and a PCR cut-off value was not used. PJP prophylaxis was defined as prescription of a prophylactic dose of co-trimoxazole for at least 2 weeks and/or aerolised pentamidine. This study was approved by the Institutional Review Board of the University of Hong Kong/Hospital Authority Hong Kong West Cluster.

\section{Statistical analysis}

Baseline characteristics of patients were compared using Student's $t$ test for continuous and the $\chi^{2}$ test between patients with and without PJP. Prevalence of PJP, prophylaxis and mortality were calculated. Number needed to treat (NNT) analysis was performed based on absolute risk reduction of PJP in patients with and without prior PJP prophylaxis.

\section{Results}

A total of 21,587 unique rheumatic patients were analysed. The mean age was $58.1 \pm 17.4$ years and the male: female ratio was 1:2.3 (Table 1). Majority of patients had RA (54\%), followed by SLE (25\%), SpA (13\%), IMM (5\%), AAV (2\%) and SSc (1\%).

Between 2015 and 2019, 1141 (5.3\%) patients were prescribed PJP prophylaxis and 48 (0.2\%) developed PJP. The majority 1109 (97.2\%) of patients were prescribed oral co-trimoxazole and $32(2.8 \%)$ were prescribed aerolised pentamidine. No patients who developed PJP were prescribed prophylaxis prior to infection. There was no 
Table 1 Baseline characteristics, frequency of PJP and prophylaxis prescription

\begin{tabular}{|c|c|c|c|c|c|c|c|}
\hline & Total & PJP & No PJP & $P$ value & Prophylaxis & No prophylaxis & $P$ value \\
\hline All patients & 21,587 & $48(0.2 \%)$ & 21,539 (99.8\%) & & 1141 (5.3\%) & 20,446 (94.7\%) & \\
\hline Age & $58.1 \pm 17.4$ & $58.5 \pm 18.4$ & $58.1 \pm 17.4$ & 0.85 & $52.8 \pm 17.7$ & $58.3 \pm 17.3$ & $<0.01$ \\
\hline Male & $5822(27.0 \%)$ & $16(33.3 \%)$ & 5806 (27.0\%) & 0.32 & $285(25.0 \%)$ & 5537 (27.1\%) & 0.12 \\
\hline Prophylaxis & 1141 (5.3\%) & 0 & 1141 (5.3\%) & 0.10 & - & - & - \\
\hline RA & 11,646 & $13(0.1 \%)$ & 11,633 (99.9\%) & & $72(0.6 \%)$ & 11,574 (99.4\%) & \\
\hline Age & $63.4 \pm 15.8$ & $68.3 \pm 17.3$ & $13.4 \pm 15.8$ & 0.27 & $63.7 \pm 14.1$ & $63.4 \pm 15.8$ & 0.89 \\
\hline Male & $2507(21.5 \%)$ & $5(38.5 \%)$ & 2502 (21.5\%) & 0.14 & $17(23.6 \%)$ & 2490 (21.5\%) & 0.67 \\
\hline Prophylaxis & $72(0.6 \%)$ & 0 & $72(0.6 \%)$ & 0.78 & - & - & - \\
\hline SLE & 5460 & $22(0.4 \%)$ & 5438 (99.6\%) & & 629 (11.5\%) & 4831 (88.5\%) & \\
\hline Age & $48.8 \pm 16.3$ & $46.8 \pm 14.2$ & $48.8 \pm 16.4$ & 0.57 & $46.4 \pm 16.6$ & $49.1 \pm 16.3$ & $<0.01$ \\
\hline Male & $551(10.1 \%)$ & 7 (31.8\%) & 544 (10.0\%) & $<0.01$ & $73(116 \%)$ & 478 (9.9\%) & 0.18 \\
\hline Prophylaxis & $629(11.5 \%)$ & 0 & 629 (11.6\%) & 0.09 & - & - & - \\
\hline SpA & 2918 & 0 & 2918 (100.0\%) & & $49(1.7 \%)$ & 2869 (98.3\%) & \\
\hline Age & $51.8 \pm 16.3$ & - & $51.8 \pm 16.3$ & - & $60.0 \pm 14.6$ & $51.7 \pm 16.3$ & $<0.01$ \\
\hline Male & 2229 (76.4\%) & - & 2229 (76.4\%) & - & 42 (85.7\%) & 2187 (76.2\%) & 0.12 \\
\hline Prophylaxis & $49(1.7 \%)$ & - & $49(1.7 \%)$ & - & - & - & - \\
\hline IMM & 1026 & $7(0.7 \%)$ & 1019 (99.3\%) & & $220(21.4 \%)$ & 806 (78.6\%) & \\
\hline Age & $61.8 \pm 15.9$ & $66.6 \pm 14.6$ & $61.8 \pm 15.9$ & 0.43 & $59.7 \pm 13.3$ & $62.4 \pm 16.5$ & 0.01 \\
\hline Male & $343(33.4 \%)$ & $2(28.6 \%)$ & 341 (33.5\%) & 0.78 & 78 (35.5\%) & 265 (32.9\%) & 0.47 \\
\hline Prophylaxis & $220(21.4 \%)$ & 0 & $220(21.6 \%)$ & 0.17 & - & - & - \\
\hline AAV & 430 & $6(1.4 \%)$ & 424 (98.6\%) & & $135(31.4 \%)$ & 295 (68.6\%) & \\
\hline Age & $65.0 \pm 17.8$ & $64.0 \pm 18.0$ & $65.0 \pm 17.8$ & 0.89 & $63.7 \pm 18.4$ & $65.6 \pm 17.5$ & 0.30 \\
\hline Male & $177(41.2 \%)$ & $2(33.3 \%)$ & 175 (41.3\%) & 0.70 & 67 (49.6\%) & $110(37.3 \%)$ & 0.02 \\
\hline Prophylaxis & $135(31.4 \%)$ & 0 & 135 (31.8\%) & 0.10 & - & - & - \\
\hline SSC & 109 & $2(1.8 \%)$ & 107 (98.2\%) & & $36(33.0 \%)$ & $73(67.0 \%)$ & \\
\hline Age & $54.4 \pm 15.9$ & $79.0 \pm 14.1$ & $54.0 \pm 15.6$ & 0.03 & $52.5 \pm 18.5$ & $55.4 \pm 14.4$ & 0.37 \\
\hline Male & $15(13.8 \%)$ & 0 & $15(14.0 \%)$ & 0.57 & $8(22.2 \%)$ & $7(9.6 \%)$ & 0.07 \\
\hline Prophylaxis & $36(33.0 \%)$ & 0 & 36 (33.6\%) & 0.32 & & & \\
\hline
\end{tabular}

PJP Pneumocystis jiroveci pneumonia; SSC systemic sclerosis, AAV ANCA-associated vasculitis; IMM immune-mediated myositis; SLE systemic lupus erythematosus; RA rheumatoid arthritis; SPA spondyloarthritis

significant association between age and PJP. Among SLE patients, male sex was associated with PJP. PJP prophylaxis was prescribed more frequently among younger patients, especially in IMM and SLE, but not in SpA (Table 1).

Among different rheumatic diseases, the risk of PJP was highest among patients with SSc, AAV, and IMM with annual incidence rates of PJP 3.6, 2.8 and 1.4 per 1,00,000 patient-year respectively. The frequency of PJP prophylaxis prescription followed a similar pattern with the highest prescription rates for patients with SSc (33.0\%), AAV (31.4\%) and IIM (21.4\%), followed by SLE (11.5\%) and RA (0.6\%) (Fig. 1).

Absolute risk reduction of PJP was calculated for each disease categories in patients with and without PJP prophylaxis. The NNT to prevent one PJP were lowest among patients with SSc (36), AAV (48) and IIM (114) (Table 2). Among the disease categories with highest PJP risk, the majority of PJP occurred at P15 or above $(100 \%$ in SSc and IIM, 66.7\% in AAV) (Fig. 2). Out of the 48 patients with PJP, there were 19 PJP-related mortalities. The mortality rate of PJP among rheumatic patients in this study was $39.6 \%$.

\section{Discussion}

Our longitudinal study of over 21,000 rheumatic patients over a 5-year period is the largest epidemiological study of PJP and PJP prophylaxis ever reported. Our comprehensive territory-wide database also allowed us to uniquely calculate the estimated annual incidence of PJP among rheumatic patients from the entire region of Hong Kong. We confirm that the risk of PJP was highest among patients with SSc, AAV and IMM; as well as to identify a "threshold" GC dose of > P15 of which the majority of PJP occurred.

In contrast to other immunocompromising conditions, the risk of PJP is relatively low for rheumatic patients. Even among patients with SSc (highest risk), the annual 


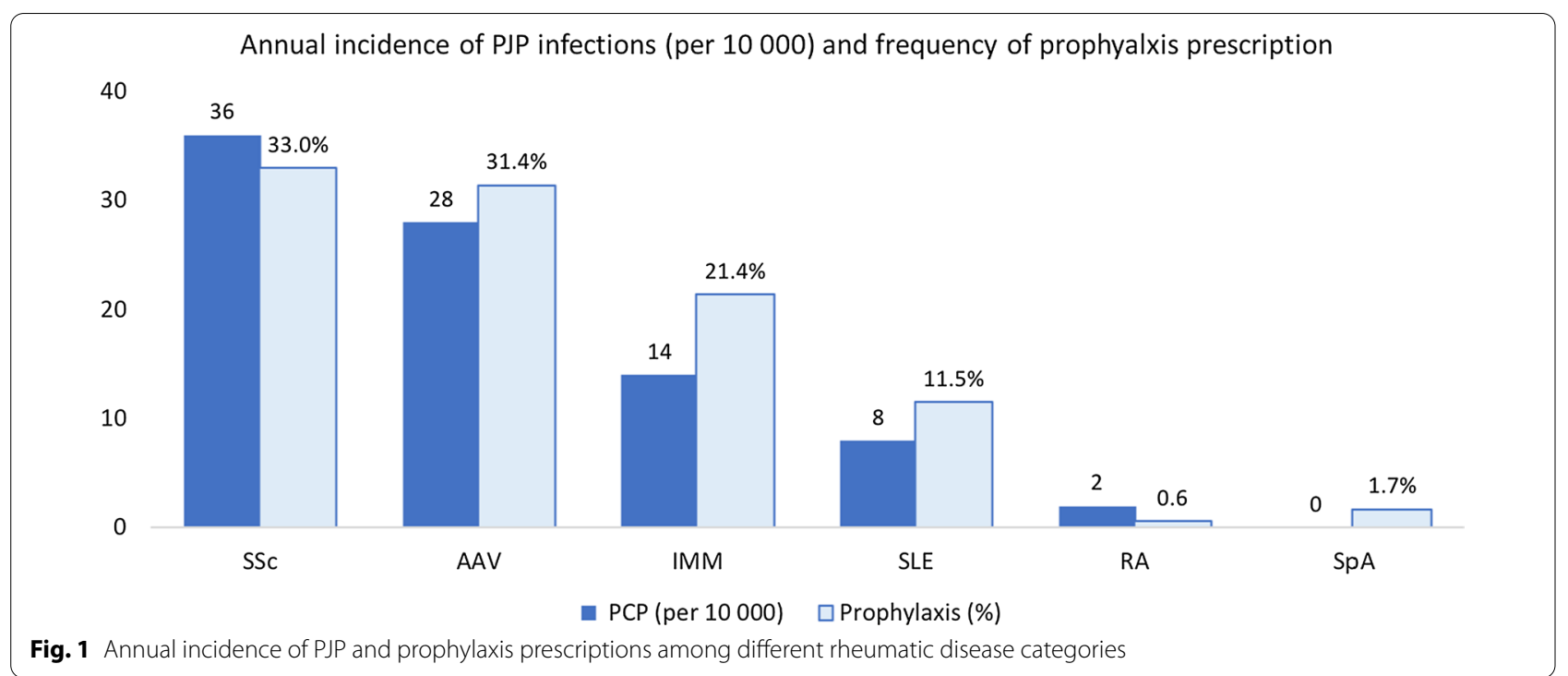

Table 2 Effectiveness of PJP prophylaxis among different rheumatic diseases

\begin{tabular}{|c|c|c|c|c|c|}
\hline Diagnosis & Total & Ever PJP & No PJP & ARR (\%) & NNT \\
\hline All patients & 21,587 & 48 & 21,540 & & 435 \\
\hline Prophylaxis & & 0 & 1142 & 0.23 & \\
\hline No prophylaxis & & 48 & 20,398 & & \\
\hline RA & 11,646 & 13 & 11,633 & & 909 \\
\hline Prophylaxis & & 0 & 72 & 0.11 & \\
\hline No prophylaxis & & 13 & 11,561 & & \\
\hline SpA & 2918 & 0 & 2918 & - & - \\
\hline Prophylaxis & & 0 & 49 & & \\
\hline No prophylaxis & & 0 & 2869 & & \\
\hline SLE & 5460 & 22 & 5438 & & 217 \\
\hline Prophylaxis & & 0 & 629 & 0.46 & \\
\hline No prophylaxis & & 22 & 4809 & & \\
\hline IMM & 1026 & 7 & 1019 & & 114 \\
\hline Prophylaxis & & 0 & 220 & 0.88 & \\
\hline No prophylaxis & & 7 & 799 & & \\
\hline AAV & 430 & 6 & 424 & & 48 \\
\hline Prophylaxis & & 0 & 135 & 2.08 & \\
\hline No prophylaxis & & 6 & 289 & & \\
\hline SSC & 109 & 2 & 107 & & 36 \\
\hline Prophylaxis & & 0 & 36 & 2.81 & \\
\hline No prophylaxis & & 2 & 71 & & \\
\hline
\end{tabular}

PJP Pneumocystis jiroveci pneumonia; SSC systemic sclerosis; AAV ANCAassociated vasculitis; IMM immune-mediated myositis; SLE systemic lupus erythematosus; $R A$ rheumatoid arthritis; $S p A$ spondyloarthritis; $A R R$ absolute risk reduction; $N N T$ number needed to treat

incidence rate was only 3.6 per $1,00,000$ patient-year, in comparison to up to 430 per $1,00,000$ patient-year in SOT patients, and 7 per 1,00,000 patient-year in HIV patients $[17,18]$. Despite a seemingly lower risk, PJP still poses a significant health burden with a mortality rate of around $40 \%$-which is higher than previous reports of PJP-related mortality of $33.3 \%$ among HIV patients in Hong Kong [19].

Our unique and comprehensive study shows that the overall risk of PJP in general rheumatic patients is low, especially compared to other immunocompromising conditions. Higher incidence rates have been previously reported in smaller studies, but those studies were mostly limited to only including hospitalized patients [20]. In contrast, our data more accurately reflects the burden of PJP across entire rheumatic populations, including both community-based and hospitalized patients. A stratified approach is important taking account of patients' factors, disease categories and immunosuppressants use to identify rheumatic patients at risk of PJP to guide the prescription PJP prophylaxis. For example, the Joint European League against Rheumatism and European Renal Association-European Dialysis and Transplant Association guideline recommended patients with AAV to receive PJP prophylaxis when treated with cyclophosphamide [21]. However, guidelines for other rheumatic diseases are currently lacking and epidemiology data, such as from this present study, would be imperative to inform these decisions.

Our study identified a "threshold" GC dose of which the majority of PJP occurred. Several mechanisms have been proposed to explain the predisposition to PJP in patients on GC. Firstly, GC reduces the numbers of circulating lymphocytes by enhancing apoptosis, including CD $4+\mathrm{T}$ cells which are essential in coordinating anti-Pneumocystis immunity [22]. Alveolar macrophages 


\section{GC dose at PJP diagnosis (median and IQR)}

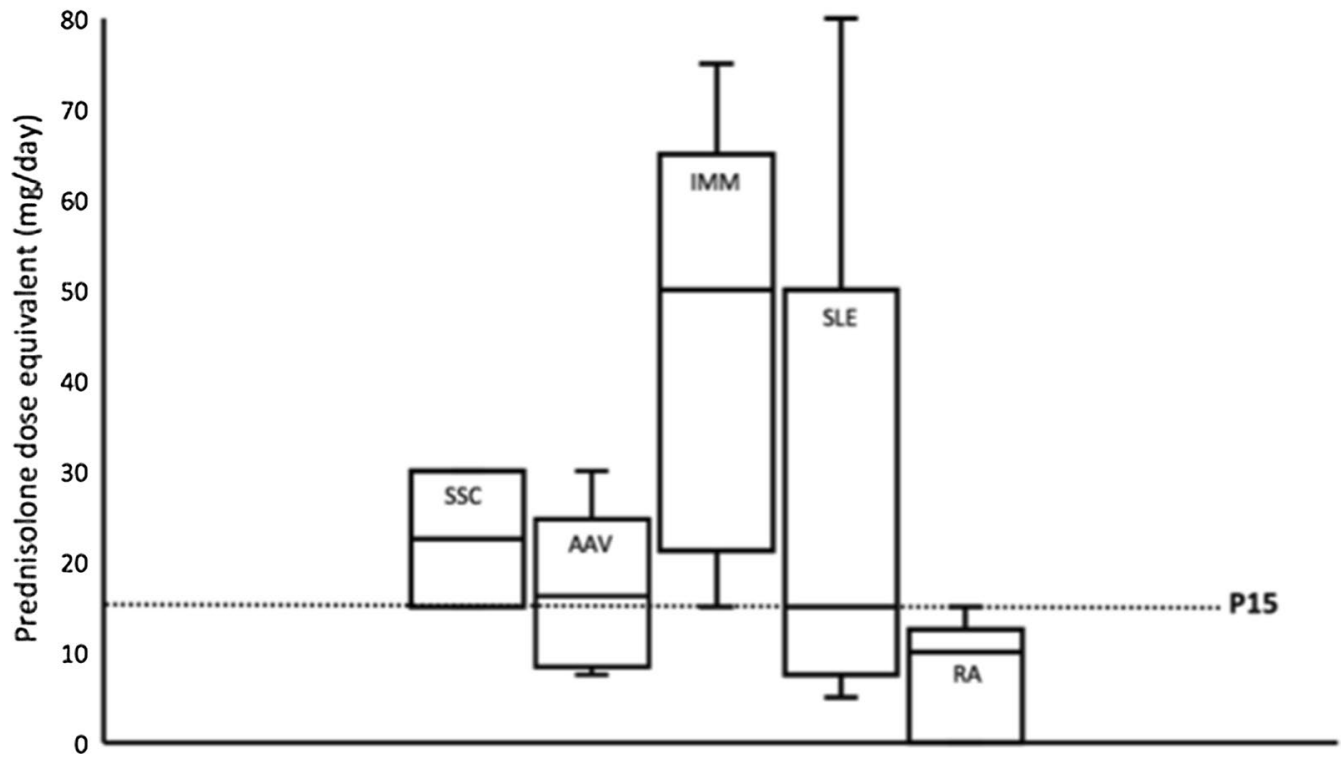

Fig. 2 Box-and-whisker plot of GC dose (median and interquartile range) at PJP diagnosis. GC glucocorticoid; PJP Pneumocystis jiroveci pneumonia; IQR interquartile range; P15 glucocorticoid at $15 \mathrm{mg}$ daily prednisolone-equivalent dose; SSC systemic sclerosis; AAV ANCA-associated vasculitis; IMM immune-mediated myositis; SLE systemic lupus erythematosus; RA rheumatoid arthritis

are the primary resident phagocytes responsible for the clearance of pneumocystis from the lung. Under the action of GC, key transcription factors such as mitogenactivated protein kinase and nuclear factor- $\mathrm{\kappa B}$ are also inhibited, leading to downregulation of pro-inflammatory cytokines secretion by alveolar macrophages [22]. In our study, most cases of PJP in the highest risk disease groups (SSc, AAV and IMM) occurred when the patients were receiving $\geq \mathrm{P} 15$, which was in line with the findings from previous studies. Although the CD4 cell count is a good marker to guide PJP prophylaxis among HIV patients, studies examining its role in non-HIV patients remain limited [8]. Further studies are needed to evaluate whether absolute lymphocyte and CD4 counts (independent of GC effect) will be useful to predict the risk of PJP in rheumatic patients.

PJP prophylaxis demonstrated extreme effectiveness in this present study, with zero cases of PJP among the 1141 (5.3\%) patients who were prescribed either oral co-trimoxazole and inhaled pentamidine. Despite its tremendous effectiveness, the potential adverse effects and risks of PJP prophylaxis must also be taken into consideration. The potential side effects of co-trimoxazole can range widely from mild to severe skin rashes (including lifethreatening reactions such as severe cutaneous adverse drug reactions), leukopenia, liver and renal dysfunction [23]. Aerolised pentamidine can cause acute respiratory reactions during inhalation [23]. According to a Korean study by Park et al., the number needed to harm was 131 for co-trimoxazole [11]. Based on the NNT calculations, we recommend that PJP prophylaxis should be initiated for SSc, AAV and IIM especially when prescribed a GC doses above P15.

Limitations of this study stem from its observational nature. Even though the prescription patterns for PJP prophylaxis correlated with the frequency of PJP in different rheumatic disease diseases, prophylaxis was prescribed according to individual physician discretion and the criteria for prophylaxis were not standardized. We were unable to investigate or control for other potential factors which may have influenced prescription decisions (such as centre-specific or physician-specific preferences). Dedicated prospective studies investigating the optimal strategies and cost-utility of PJP prophylaxis are underway. Furthermore, despite being a very comprehensive study of all patients in the public sector, a minority ( $<10 \%$ in Hong Kong) of patients might receive medical care solely from private physicians and have been missed in the current study. Additionally, although this study evaluated the risk-benefit ratio of PJP prophylaxis across different rheumatic disease categories, further studies for cost-effectiveness would be useful. However, the absolute cost of oral cotrimoxazole at standard prophylaxis dose in Hong Kong costs less than USD 1 dollar per week and adverse drug reactions remain uncommon. The cost of prophylaxis would unlikely contribute to significant cost burden, especially in comparison 
to the cost of healthcare utilization and productivity loss associated with PJP. However, further local studies on potential harm of PJP prophylaxis and number needed to treat analysis (especially on the use of aerolised pentamidine in the era of COVID) are urgently needed.

\section{Conclusion}

In conclusion, PJP is an uncommon but important infection among rheumatic patients with high mortality. PJP prophylaxis is effective and should be considered in patients with SSc, AAV and IMM, especially in those receiving glucocorticoid doses above P15.

\begin{abstract}
Abbreviations
AAV: Anti-neutrophil cytoplasmic antibody-associated vasculitis; CYC: Cyclophosphamide; GC: Glucocorticoids; HA: Hospital authority; HIV: Human immunodeficiency virus; ICD-9: International Classification of Diseases, Ninth Revision; IMM: Immune-mediated myositis; NNT: Number needed to treat; OR: Odds ratio; P: Prednisolone; PJP: Pneumocystis jiroveci pneumonia; RA: Rheumatoid arthritis; SLE: Systemic lupus erythematosus; SOT: Solid organ transplant; SpA: Spondyloarthritis; SSc: Systemic sclerosis.
\end{abstract}

\section{Acknowledgements}

We thank Dr. Shirley X. Li (Assistant Professor, Department of Medicine, University of Hong Kong) for her help with statistical analysis and input.

\section{Authors' contributions}

SCWC was responsible for methodology, data analysis and writing (original draft). HYC was responsible for conceptualization and methodology. CSL was responsible for conceptualization and writing (review and editing). PHL was responsible for methodology, data analysis and writing (review and editing). All authors read and approved the final manuscript.

\section{Funding}

There was no funding for this work.

\section{Availability of data and materials}

The datasets used and/or analyzed during the current study are available from the corresponding author on reasonable request.

\section{Declarations}

\section{Ethics approval and consent to participate}

This study was approved by the institutional review board of the University of Hong Kong/Hospital Authority Hong Kong West Cluster (Institutional Review Board Reference Number: UW20-808). Consent was exempted by the ethics review board on the basis of anonymous retrospective data collection.

\section{Consent for publication}

All authors concur with the submission and accept the transfer of the copyright to the publisher upon acceptance.

\section{Competing interests}

The authors declare that they have no competing interests.

Received: 3 August 2021 Accepted: 29 October 2021

Published online: 11 November 2021

\section{References}

1. Grimwade K, Swingler G. Cotrimoxazole prophylaxis for opportunistic infections in children with HIV infection. Cochrane Database Syst Rev. 2003. https://doi.org/10.1002/14651858.CD003508.
2. Kaplan JE, Hanson D, Dworkin MS, Frederick T, Bertolli J, Lindegren $M L$, et al. Epidemiology of human immunodeficiency virus-associated opportunistic infections in the United States in the era of highly active antiretroviral therapy. Clin Infect Dis. 2000;30(Suppl 1):S5-14.

3. Morris A, Lundgren JD, Masur H, Walzer PD, Hanson DL, Frederick T, et al. Current epidemiology of Pneumocystis pneumonia. Emerg Infect Dis. 2004;10(10):1713-20

4. Mansharamani NG, Garland R, Delaney D, Koziel H. Management and outcome patterns for adult Pneumocystis carinii pneumonia, 1985 to 1995: comparison of HIV-associated cases to other immunocompromised states. Chest. 2000;118(3):704-11.

5. Taplitz RA, Kennedy EB, Bow EJ, Crews J, Gleason C, Hawley DK, et al. Antimicrobial prophylaxis for adult patients with cancer-related immunosuppression: ASCO and IDSA clinical practice guideline update. J Clin Oncol. 2018;36(30):3043-54.

6. Fishman JA, Gans H, Practice ASTIDCo. Pneumocystis jiroveci in solid organ transplantation: guidelines from the American Society of Transplantation Infectious Diseases Community of Practice. Clin Transplant. 2019;33(9):e13587.

7. Tomblyn M, Chiller T, Einsele H, Gress R, Sepkowitz K, Storek J, et al. Guidelines for preventing infectious complications among hematopoietic cell transplantation recipients: a global perspective. Biol Blood Marrow Transplant. 2009;15(10):1143-238.

8. HIV Manual. Department of Health, Government of the HKSAR, Hong Kong; 2006.

9. Ward MM, Donald F. Pneumocystis carinii pneumonia in patients with connective tissue diseases: the role of hospital experience in diagnosis and mortality. Arthritis Rheum. 1999;42(4):780-9.

10. Mecoli CA, Saylor D, Gelber AC, Christopher-Stine L. Pneumocystis jiroveci pneumonia in rheumatic disease: a 20-year single-centre experience. Clin Exp Rheumatol. 2017;35(4):671-3.

11. Park JW, Curtis JR, Moon J, Song YW, Kim S, Lee EB. Prophylactic effect of trimethoprim-sulfamethoxazole for Pneumocystis pneumonia in patients with rheumatic diseases exposed to prolonged high-dose glucocorticoids. Ann Rheum Dis. 2018;77(5):644-9.

12. Park JW, Curtis JR, Kim MJ, Lee H, Song YW, Lee EB. Pneumocystis pneumonia in patients with rheumatic diseases receiving prolonged, nonhigh-dose steroids-clinical implication of primary prophylaxis using trimethoprim-sulfamethoxazole. Arthritis Res Ther. 2019;21(1):207.

13. Lau WCY, Cheung CL, Man KKC, Chan EW, Sing CW, Lip GYH, et al. Association between treatment with apixaban, dabigatran, rivaroxaban, or warfarin and risk for osteoporotic fractures among patients with atrial fibrillation: a population-based cohort study. Ann Intern Med. 2020;173(1):1-9.

14. Leung WK, Wong IOL, Cheung KS, Yeung KF, Chan EW, Wong AYS, et al. Effects of helicobacter pylori treatment on incidence of gastric cancer in older individuals. Gastroenterology. 2018;155(1):67-75.

15. Lau WC, Chan EW, Cheung CL, Sing CW, Man KK, Lip GY, et al, Association between dabigatran vs warfarin and risk of osteoporotic fractures among patients with nonvalvular atrial fibrillation. JAMA. 2017;317(11):1151-8.

16. Wong AY, Root A, Douglas IJ, Chui CS, Chan EW, GhebremichaelWeldeselassie Y, et al. Cardiovascular outcomes associated with use of clarithromycin: population based study. BMJ. 2016;352:h6926.

17. Iriart X, Bouar ML, Kamar N, Berry A. Pneumocystis pneumonia in solidorgan transplant recipients. J Fungi. 2015;1(3):293-331.

18. Buchacz K, Lau B, Jing Y, Bosch R, Abraham AG, Gill MJ, et al. Incidence of AIDS-defining opportunistic infections in a multicohort analysis of HIV-infected persons in the United States and Canada, 2000-2010. J Infect Dis. 2016;214(6):862-72.

19. Hui M, KwokWT. Pneumocystis carinii pneumonia in Hong Kong: a 10 year retrospective study. J Med Microbiol. 2006;55(Pt 1):85-8.

20. Falagas ME, Manta KG, Betsi GI, Pappas G. Infection-related morbidity and mortality in patients with connective tissue diseases: a systematic review. Clin Rheumatol. 2007;26(5):663-70.

21. Yates M, Watts RA, Bajema IM, Cid MC, Crestani B, Hauser T, et al. EULAR/ERA-EDTA recommendations for the management of ANCAassociated vasculitis. Ann Rheum Dis. 2016;75(9):1583-94.

22. Thomas CF Jr, Limper AH. Current insights into the biology and pathogenesis of Pneumocystis pneumonia. Nat Rev Microbiol. 2007;5(4):298-308 
23. Stern A, Green H, Paul M, Vidal L, Leibovici L. Prophylaxis for Pneumocystis pneumonia (PCP) in non-HIV immunocompromised patients. Cochrane Database Syst Rev. 2014. https://doi.org/10.1002/14651858. CD005590.pub3.

\section{Publisher's Note}

Springer Nature remains neutral with regard to jurisdictional claims in published maps and institutional affiliations.
Ready to submit your research? Choose BMC and benefit from:

- fast, convenient online submission

- thorough peer review by experienced researchers in your field

- rapid publication on acceptance

- support for research data, including large and complex data types

- gold Open Access which fosters wider collaboration and increased citations

- maximum visibility for your research: over $100 \mathrm{M}$ website views per year

At BMC, research is always in progress.

Learn more biomedcentral.com/submissions 